Groys, Boris (1999) Über das Neue. Versuch einer Kulturökonomie. Frankfurt a.M.: Suhrkamp.

Heifiz, I. (1951) Die Arbeit an dem Dokumentarfilm. In: Der Morgen (Berlin), 15.09.1951.

Hoffmann, Kay (2005) Menschen, Tiere, Sensationen. Die Wochenschauen der 30er Jahre. In: Zimmermann/Hoffmann 2005a, S. 211-230.

Hütt, Wolfgang (1979) Aufbruch und Selbstbestimmung. In: Weggefährten - Zeitgenossen. Zentrum für Kunstausstellungen der DDR. Hg. v. Fritz Donner. Berlin, S. 37-50

Jordan, Günter (2000) Die frühen Jahre. 1946 bis 1952. In: Schwarzweiß und Farbe. DEFA-Dokumentarfilme 1946-92. Red. Günter Jordan \& Ralf Schenk. 2. Aufl. Berlin: Jovis, S. 14-47.

Kastelin, N.A. (1953) Wie schaffen wir Dokumentarfilme? In: Tägliche Rundschau (Berlin), 12.11.1953.

Kreimeier, Klaus / Ehmann, Antje / Goergen, Jeanpaul (Hg.) (2005) Geschichte des dokumentarischen Films in Deutschland, Band 2: Weimarer Republik, 1918-1933. Stuttgart: Reclam.

Maetzig, Kurt (1946) Vom Wesen des Dokumentarfilms. In: Theater der Zeit, 1, S. 24

Mückenberger, Christiane (2000) Andrew Thorndike, Hans Speidel, Annelie Thorndike. In: Der geteilte Himmel. Arbeit, Alltag und Geschichte im ost-und westdeutschen Film. Hg. v. Peter Zimmermann \& Gebhard Moldenhauer. Konstanz: UVK.

Müller, Heiner (1994a) Geschichten aus der Produktion I. Berlin: Rotbuch.

Müller, Heiner (1994b) Bericht vom Anfang [1950]. In: Müller 1994a, S. 11-13.

Müller, Heiner (1994c) Die Korrektur [1957]. In: Müller 1994a, S. 47-58.

Nora, Pierre (1990) Zwischen Gedächtnis und Geschichte. Frankfurt a.M.: Fischer.

Pieck, Wilhelm (1946) Um die Erneuerung unserer deutschen Kultur. In: Wilhelm Pieck / Anton Ackermann: Unsere kulturpolitische Sendung: Reden auf der 1. Zentralen Kulturtagung der Kommunistischen Partei Deutschlands vom 3. - 5. Februar 1946. Berlin: Neuer Weg, S. 5-24.

Schitty, Dagmar (2002) Zwischen Regie und Regime. Filmpolitik der SED im Spiegel der DEFA-Produktionen. Berlin: Links.

Träger, Claus (Hg.) (1986) Wörterbuch der Literaturwissenschaft. Leipzig: Bibliographisches Institut.

Ulbricht, Walter (1979) Der Künstler im Zweijahrplan. Diskussionsrede auf der Arbeitstagung der Genossen Schriftsteller und Künstler [1948]. In: Zur Tradition der deutschen sozialistischen Literatur: Eine Auswahl von Dokumenten. Hg. v. Friedrich Albrecht. Berlin: Aufbau, S. 436-439.

Varga, Lucie (1991) Zeitenwende. Mentalitätshistorische Studien 1936-1939. Hg., übers. u. eingel. v. Peter Schöttler. Frankfurt a.M.: Suhrkamp.

Weidhaas, Volker (1979) DEFA-Wochenschauen 1946-49 in der Akademie der Künste. In: Forum (Berlin), 29.10.1979.

Zimmermann, Peter (2005a) Im Banne der Ufa-Ästhetik und des ‘Kalten Krieges». Film- und Fernseh-Dokumentationen der BRD und DDR über das «Dritte Reich : In: Zimmermann/Hoffmann 2005a, S. 710-719.

Zimmermann, Peter (2005b) Kontinuitäten und Wandlungen im Zeichen von «Entnazifizierung) und 〈Reeducation〉. In: Zimmermann/Hoffmann 2005a, S. 691-709.

Zimmermann, Peter / Hoffmann, Kay (Hg.) (2005a) Geschichte des dokumentarischen Films in Deutschland. Band 3: "Drittes Reich», 1933-1945. Stuttgart: Reclam.

Zimmermann, Peter / Hoffmann, Kay (2005b) Die Kulturfilm-Debatte zur Zeit des Nationalsozialismus und die Rechtfertigungsliteratur nach 1945. In: Zimmermann/Hoffmann 2005a, S. 26-32.
Judith Keilbach

\section{Archive sagen aus \\ Zum Stellenwert von Filmdokumenten in den Filmen von Andrew und Annelie Thorndike}

Die Auseinandersetzung mit der nationalsozialistischen Vergangenheit war für die DDR von großer Bedeutung. Für ihr Selbstverständnis als antifaschistischer Staat galt es, sich von dieser Vergangenheit deutlich abzugrenzen und die Bevölkerung über die Ursachen und Triebkräfte des Nationalsozialismus oder Faschismus ${ }^{1}$ aufzuklären, um eine Wiederholung zu verhindern. Diese Aufklärungsarbeit fand nicht zuletzt auch im Bereich der Kulturpolitik statt, in deren Rahmen Spielfilme wie Rat Der Götter (DDR 1950, Kurt Maetzig), Professor MaMlock (DDR 1961, Konrad Wolf) und NACKT UNTER WöLFEN (DDR 1963, Frank Beyer), Fernsehserien wie Gewissen IN Aufruhr (DDR 1961, Günter Reisch/ Hans-Joachim Kasprzik) und JeDER STIRbT Für SICH AlLEIN (DDR 1970, Hans-Joachim Kasprzik) sowie zahlreiche Dokumentarfilme entstanden sind.

In den 1950er Jahren waren es vor allem Andrew und Annelie Thorndike, die sich der dokumentarischen Auseinandersetzung mit dem Nationalsozialismus verschrieben hatten. In ihren Filmen DU UND MANCHER KaMERAD (DDR 1956), URLAUB AUF SYLT (DDR 1957) und UNTERNEHMEN TEUTONENSCHWERT (DDR 1958) blicken sie jedoch nicht nur auf die deutsche Geschichte zurück, sondern beziehen auch die Gegenwart beider deutscher Staaten mit ein. Sie verstehen den Faschismus als Folge des Kapitalismus, dessen Wachstumsorientierung zwangsläufig zum Krieg führe, und warnen vor einem wiederauflebenden Militarismus in der $\mathrm{BRD}$, der aus der personellen Kontinuität der Machteliten in Politik, Militär und Wirtschaft resultiere. ${ }^{2}$ Die Filme greifen dabei auf unterschiedliche argumentative Verfahren zurück: Während Du und MANCHER KAMERAD vor allem Thesen zum Faschismus und zur deutsch-deutschen

1 Bezieht sich der Begriff «Nationalsozialismus» auf das 'Dritte Reich', so beschreibt Fenr viel breiteres ideologisches und institutionelles Feld. Während Faschis in ches) implizierte, spielt dieser Aspekt in aktuellen Faschismustheorien nur noch eine untergeordnete Rolle.

2 Die Warnung vor einem drohenden Krieg ist nicht zuletzt im Kontext der Gründung der Bundeswehr und des Beitritts der BRD zur NATO zu sehen. 
Gegenwart vorstellt, versuchen URLAUb aUf SYLT und UNTERNEHMEN Teutonenschwert «an zwei [...] Einzelbeispielen das Weiterleben des Faschismus in der Bundesrepublik nach[zuweisen]» (Roth 1977, 170). Den Filmen lässt sich die Verwendung «unzulässige[r] Hilfsmittel» (nicht ausgewiesene Nachinszenierungen) sowie das Verschweigen historischer Fakten (deutsch-sowjetischer Nichtangriffspakt) und Personen (Stalin) vorwerfen (ebd., 171). Auch der «Schematismus der Beweisführung» und die nicht belegten Schlussfolgerungen sind in die Kritik geraten (ebd., 172).

Der vorliegende Text wird weder diese (berechtigten) Kritikpunkte weiterverfolgen noch aus einer historischen Perspektive eine ausführliche Kontextualisierung der Filme vornehmen (vgl. hierzu wiederum Roth 1977; Steinle 2003, Heimann 1996). Das Interesse hier ist vielmehr ein filmwissenschaftliches und beschränkt auf den Einsatz des Bildmaterials, das in verschiedenen Filmarchiven recherchiert und dann der Argumentation der Filme entsprechend kompiliert wurde. Anhand von Beispielen soll die Methode der Montage genauer erläutert und der Stellenwert der Bilder als Beweismaterial diskutiert werden.

\section{Andrew und Annelie Thorndike}

Andrew Thorndike (1909-1979) hatte vor seiner Tätigkeit bei der DEFA bereits Filme für die Ufa gedreht. Zunächst war er in der Werbefilmabteilung als Generalvertreter für die Betreuung von Werbekunden zuständig; ab 1941 stellte er für das Oberkommando der Kriegsmarine und des Heeres selbst Lehrfilme her und drehte 1942 den Kulturfilm Dre Herrin DES HOFEs. Dieser Film stellt den Alltag einer idealtypischen Bäuerin (die allerdings von einer Schauspielerin verkörpert wurde) dar und zeigt, wie diese ihre Arbeit auf dem Hof und im Haushalt verrichtet, das Gesinde überwacht, ihre Kinder versorgt und ihrem Sohn einen Brief ins HJ-Lager schreibt. Thorndikes Fassung stieß jedoch auf Kritik, so dass der Film erst in einer umgearbeiteten Version, die mit dem Prädikat (volksbildend) ausgezeichnet wurde, in die Kinos kam. Im selben Jahr wurde Andrew Thorndike unter dem Verdacht der Wehrkraftzersetzung verhaftet und später zur Wehrmacht einberufen. Nachdem er aus der russischen Kriegsgefangenschaft entlassen wurde, wo er dem Nationalkomitee ‘Freies Deutschland beigetreten war, begann er 1949 für das «DEFA-Studio für Wochenschau und Dokumentarfilme> zu arbeiten. Da in der Sowjetischen Besatzungszone eine unbelastete Vergangenheit Voraussetzung war, um beim Film arbeiten zu können, schienen Thorndikes antifaschistisches
Engagement in der Kriegsgefangenschaft und sein Wunsch, beim Aufbau einer sozialistischen Gesellschaft mitwirken zu können, die verantwortlichen Funktionäre ausreichend überzeugt zu haben, so dass er - trotz der früheren Tätigkeit bei der Ufa - seinen Beruf als Regisseur fortsetzen konnte. Rückblickend beschreibt Thorndike diesen Neubeginn folgendermaßen:

[...] Gewandelt durch das furchtbare Erlebnis des Krieges, durch die Begegnung mit Sowjetmenschen und die jahrelange Beschäftigung mit der marxistisch-leninistischen Theorie, [wollte ich] im Sinne des sozialistischen Ideals handeln [...] (Thorndike, zit. n. www.filmportal.de, Andrew Thorndike).

Andrew Thorndikes frühe DEFA-Filme demonstrieren seinen festen Glauben an eine neue Gesellschaft: In seinem ersten feierte er den Bergarbeiter Adolf Hennecke, der am 13. Oktober 1948 im Steinkohleabbaugebiet Zwickau in einer Sonderschicht das Fördersoll mit 380 Prozent übererfüllte. Mit dieser Leistung legte Hennecke nicht nur Enthusiasmus für den Aufbau eines neuen Deutschland an den Tag, er begründete damit auch die Aktivistenbewegung zur Steigerung der Arbeitsproduktivität. Thorndike begnügte sich allerdings nicht mit der Darstellung des Beginns dieser Bewegung: Er inszenierte zusätzliche Passagen, um die neue Gesellschaftsordnung in Ostdeutschland dem Fortbestehen der alten, kapitalistischen Machtverhältnisse in Westdeutschland gegenüberzustellen, wobei in diesen Spielszenen auch die Figur eines WestIndustriellen und Hugenberg-Freunds auftritt, die sich als Anspielung auf Thorndikes Vater verstehen lässt (und mit der sich der Regisseur von seiner großbürgerlichen Vergangenheit distanziert). Nach heftiger Kritik an den Nachinszenierungen wurde DER DURCHBROCHENE KREIS (so der ursprüngliche Titel) allerdings umgearbeitet und schließlich als 15-minütiger Dokumentarfilm unter dem Titel Adolf HeNNECKE bzw. als Der 13. OKтовеR (DDR 1949) veröffentlicht. Auch seine folgenden Filme rühmten die Leistungen Einzelner oder des Kollektivs, die Ostdeutschland kurz nach dem Krieg wieder zum Blühen brächten. Dass dieses Bild nicht der tatsächlichen Situation entsprach, gab Thorndike rückblickend freimütig zu, denn in den Aufbaujahren wäre eine Zustandsbeschreibung, so der Regisseur, «traurig ausgefallen» (zit. n. Steinle 2003, 51). So dokumentieren die Filme anstelle der historischen Realität eher eine Wunschvorstellung: In Von Hamburg BIS STRALSUND (DDR 1950) zeigt Thorndike die Aufbauleistungen in den volkseigenen Werften der DDR und kontrastiert diese mit den brachliegenden Häfen der BRD. In DER WEG NACH OBEN (DDR 1950, Andrew Thorndike/Karl Gass) schildert er den erfolgreichen 
Aufstieg der DDR, indem er die wichtigsten Daten und Leistungen seit 1945 zusammenfasst. ${ }^{3}$

Annelie Thorndike (geb. 1925) war Lehrerin, bevor sie zum Film kam. Auch sie beteiligte sich enthusiastisch am Aufbau einer neuen Gesellschaft und gründete 1948 im ländlichen Mecklenburg die erste Zentralschule, für die sie als Aktivistin ausgezeichnet wurde. Bei einem Besuch der Schule im Rahmen der Recherche für DER WEG NACH OBEN lernten sich die beiden kennen. Der Film Die Prüfung (DDR 1952, Andrew Thorndike), der einen Einblick in das Schulsystem und die pädagogischen Vorstellungen der DDR gibt, war die erste filmische Zusammenarbeit des Paares. Für diesen ersten gemeinsamen Thorndike-Film schrieb Annelie das Drehbuch, während Andrew Regie führte. Im Gegensatz zu dieser klaren Arbeitstrennung ist in den Produktionsangaben der nachfolgenden Filme keine eindeutige Aufgabenverteilung mehr nachgewiesen. Dennoch gab es getrennte Arbeitsbereiche: Annelies Neigung, so Andrew Thorndike, gehörte «mehr dem Wort [...], der literarischen Seite, dem Kommentar, der Arbeit mit dem Ton, der Synchronisation», während er für die Montage und Musik zuständig war. «Es hat so etwas wie eine Spezialisierung eingesetzt, die natürlich von Fall zu Fall Veränderungen und Verschiebungen zeigt», beschreibt Andrew Thorndike (1982, zit. n. www.filmportal.de, Andrew Thorndike) die Arbeitsweise des Paares, die die Kooperation in den DEFA-Produktionsgruppen insgesamt prägte. Die Thorndikes arbeiteten in einem Produktionskollektiv ( Gruppe Thorndike> bzw. ‘DEFA-Gruppe 67^) mit einem festen Stamm von Mitarbeitern, die häufig als Co-Autoren oder Co-Regisseure der ‘Thorndike-Filme` fungierten und diese maßgeblich mitgestalteten. ${ }^{4}$

Mit ihrer Arbeit prägten die Thorndikes einen wesentlichen Bereich der Filmkultur und hatten Einfluss auf zahlreiche Dokumentaristen der DDR. Insbesondere ihre Verwendung von Archivmaterial als Beleg für die NaziVergangenheit von führenden westdeutschen Politikern und Offizieren wurde von anderen Filmemachern aufgegriffen: Beispielsweise wies Walter Heynowski in seinen Fernsehdokumentationen MORD IN Lwow (DDR 1959) und AKrION J (DDR 1961) die Beteiligung von Theodor Oberländer (1953-1960 Bundesminister für Vertriebene, Flüchtlinge und Kriegsgeschädigte) und von Hans Globke (1953-1963 Staatssekretär im Bundeskanzleramt) an NS-Verbrechen nach; und Joachim Hellwig montierte in So MACHT MAN KANZLER (DDR 1961) Filmaufnahmen von Hitler und Adenauer so, dass sich ihre politischen Karrieren zu entsprechen scheinen. ${ }^{5}$

3 Für eine ausführliche Analyse dieses Films vgl. Steinle 2003, $49 \mathrm{ff}$.

4 Z.B. Karl-Eduard von Schnitzler, Klaus Alde und Walter Fuchs.

5 Vgl. zur weiteren Entwicklung dieser Regisseure auch die Aufsätze von Claudia Bött-
Neben Filmen, die (Wunsch-)Bilder von der DDR entwarfen und damit ein sozialistisches Gesellschaftsideal propagierten, sowie Kompilationsfilmen über den Nationalsozialismus und sein Nachwirken in der BRD drehten die Thorndikes unter anderem auch Filme und Fernsehsendungen über die Sowjetunion (DAS RUSSISCHE WUNDER [DDR 1963, Andrew Thorndike/Annelie Thorndike/Klaus Alde/Manfred Krause/ Richard Cohn-Vossen], Die Konzessionen Des Mister Urquhart [DDR 1961], Die Weichen SIND Gestellt [DDR 1965, Klaus Alde]). Einige ihrer Produktionen zeichneten sich dabei durch ein hohes innovatives Potenzial aus, was sich nicht zuletzt auch am Einsatz neuer technischer Mittel ablesen lässt: So verwendeten sie für DIE SIEBEN vom RHEIN (DDR 1954, Andrew \& Annelie Thorndike), der den Besuch von sieben westdeutschen Arbeitern im ostdeutschen Stahl- und Walzwerk Riesa dokumentiert, bereits 1954 Originaltonaufnahmen ${ }^{6}$ und drehten mit Du BIST MIN. EIN DEUTSCHES TAGEBUCH (DDR 1968/69, Hans-Joachim Funk/Manfred Kruse/Andrew \& Annelie Thorndike/Michael Englberger) einen der ersten 70-mm-Filme der DDR (vgl. Vonau 2002, 431ff). Dieser Film, der auf Tagebüchern von Annelie Thorndike basiert und in dem ein poetischer Text über die DDR mit «eindrucksvollen Landschaftsaufnahmen und spektakulären Flugaufnahmen ${ }^{7}$ unterlegt wird, kam 1969 allerdings erst nach starken Kürzungen in die Kinos. Neben diesem Einsatz von technischen Neuerungen stellte in den 1950er Jahren auch die Kompilation von historischem Bildmaterial und die Transformation von Filmaufnahmen in Beweismittel eine ungewöhnliche Methode dar, die vor allem in den Filmen der Reihe ARCHIVE SAGEN AUS systematisch zur Anwendung kam.

\section{Kompilationsfilme}

Bereits im schon erwähnten DeR WEG NACH OBEN wie auch in WILHELM PIECK - DAs Leben UNSERES PRÄSIDENTEN (DDR 1951, Andrew Thorndike) werden Aufnahmen verwendet, die nicht extra für diese Filme, sondern in anderen Kontexten entstanden sind. DER WEG NACH OBEN enthält neben neu gedrehten Bildern zahlreiche Aufnahmen aus ost- und westdeutschen Wochenschauen der vorangegangenen fünf Jahre, und in WILFELM PIECK

cher und Corinna Schier sowie von Tobias Ebbrecht im vorliegenden Band.

6 Eine Analyse des Films sowie eine Beschreibung der schwerfälligen Technik und ihrer Effekte findet sich in Steinle 2003, 74ff.

7 Zit. n. dem Programmtext zur Filmreihe Eine Frage des Formats: DEFA 70, Kino arsenal (Berlin), Juli 2006. 
sind Archivbilder aus der Geschichte der Arbeiterbewegung zu sehen, die bis auf das Jahr 1915 zurückgehen. Für ihre Filme über den Nationalsozialismus führten die Thorndikes diesen Einsatz von Archivaufnahmen systematisch fort. So entstand DU UND MANCHER KAMERAD, der die deutsche Geschichte auf die Ursachen der beiden Weltkriege befragt und im Kapitalinteresse einflussreicher Industrieller die Antwort findet, nach einer zweijährigen Recherche- und Sichtungsphase in den Filmarchiven in Ost-Berlin, Warschau, London, Paris, Rom, Prag und Moskau. ${ }^{8}$ Andrew und Annelie Thorndike sichteten in dieser Zeit rund sechs Millionen Meter Filmmaterial, wobei es sich vor allem um Wochenschauen und Dokumentarfilme der UFA handelte (vgl. Leyda 1964, 83). Nach einer ersten Vorauswahl blieben ca. 1,5 Mio. Meter übrig, von denen ca. 2850 Meter (entspricht 103 min.) zur Kompilation von DU UND MANCHER KAMERAD genutzt wurden. Auf diesen Materialfundus gehen auch die Filme aus der Reihe ARCHIVE SAGEN AUS zurück.

Die Kompilation von historischem Bildmaterial war damals - anders als in der gegenwärtigen Film- und Fernsehproduktion - kein alltägliches Verfahren. Heute lässt sich der Einsatz von Archivaufnahmen in den unterschiedlichsten Bereichen beobachten, wobei den Bildern jeweils spezifische Funktionen zukommen: In Geschichtsdokumentationen dienen historische Aufnahmen beispielsweise zur Visualisierung von Vergangenheit und versprechen dabei nicht selten einen unmittelbaren Zugang zur Geschichte; in anderen Fällen werden solche Bilder hingegen als Versatzstücke eines «Retro-Styles» verwendet und signifizieren Geschichtlichkeit oder auch Vergangenheit, ohne diese genauer zu spezifizieren. ${ }^{9}$ Wie in den aktuellen Dokumentationen über den Nationalsozialismus wird auch in den Filmen der Thorndikes die Referenzialität der Aufnahmen akzentu-

8 Heimann $(1996,73)$ und Steinle $(2003,114)$ weisen darauf hin, dass der Planungsbeginn von DU UND MANCHER KAMERAD zeitlich mit der Gründung des Filmarchivs der DDR und der Rückgabe von durch die UdSSR beschlagnahmten Filmbeständen aus dem Dritten Reich zusammenfiel, und schlagen damit implizit vor, den Film auch im Kontext der Verfügbarkeit seiner Materialgrundlage zu diskutieren.

9 William Wees (1992) unterteilt die unterschiedlichen Verwendungsweisen von Archivbildern in drei (idealtypische) Kategorien: Als Methode der Kompilation von Archivin Dokumentarfilmen anzutreffen ist, bezeichnet er einen die historische Realität vutrer künsterisch-revolutionän künstlerisch-revolutionären Praxis der 1920er Jahre verankert und heute vor allem im Avantgardefilm verortet, akzentuiert hingegen eine kritische Wahrnehmung der filmischen Darstellung und trägt zu deren Dekonstruktion bei. Als Aneignung versteht Wees hingegen eine unkritische Verwendung von Bildern, die sich nicht für deren historisch spezifischen Ursprung interessiert, sondern diese - ganz im Sinne des Postmodernismus - als reine Oberfläche nutzt. In dieser Weise verwenden beispielsweise Werbe- und Musikclips historische Aufnahmen. Sie dienen vier vor beispielsweise Grundstimmung zu erzeugen und Assoziationen freizusetzen. iert. Dabei geht es jedoch nicht nur um die Darstellung von historischer Realität. Zentrales Merkmal von Thorndikes filmischer Auseinandersetzung mit der Vergangenheit ist vielmehr die juristische Verwendung der Bilder: Die Archivaufnahmen werden als Beweismittel eingesetzt, um einzelnen Personen eine Beteiligung an nationalsozialistischen Verbrechen nachzuweisen. Diese Funktion der Archivaufnahmen stellt einen entscheidenden Unterschied zwischen den Filmen der Thorndikes und anderen Kompilationsfilmen dar.

Obwohl schon früh in der Filmgeschichte Aufnahmen aus unterschiedlichen Quellen kompiliert wurden, um aus ihnen Biografien, Jahresrückblicke oder andere Erinnerungsfilme zusammenzustellen (Leyda 1964, 18f), gilt Der Fall Der DyNaStIe Romanow (UdSSR 1927) von Esfir Shub als erster «richtiger` Kompilationsfilm. Shub war Cutterin von Spielfilmen, bevor sie die Gelegenheit erhielt, zum zehnten Jahrestag der Russischen Revolution einen eigenen Film zu produzieren, der ausschließlich aus vorrevolutionären Aufnahmen bestand. Da Filme damals nicht systematisch archiviert wurden, war es zunächst schwierig, überhaupt dokumentarische Aufnahmen aus der Zeit vor der Revolution zu finden. Doch Shub hatte das Glück, bei ihrer Suche neben alten Wochenschauen auch auf die privaten Heim- und Familienfilme des Zaren zu stoßen. Organisationsprinzip ihrer Montage war die Akzentuierung des Kontrasts zwischen dem Leben der herrschenden Elite und dem der 'normalen` Bevölkerung, indem sie beispielsweise die feiernden Romanows mit der Aufnahme eines schwer arbeitenden Bauern verbindet. ${ }^{10}$

Diese Kombination von Einstellungen ist im Rahmen der Experimente und theoretischen Überlegungen zur Montage zu situieren, die den sowjetischen Film jener Zeit prägten. Lew Kuleschow experimentierte damals beispielsweise mit unterschiedlichen Semantisierungsmöglichkeiten einzelner Einstellungen, und Sergej Eisenstein erforschte künstlerische Umsetzungen des dialektischen Prinzips, das er in der Form des Konflikts verkörpert sah und für das ihm die filmische Montage als besonders geeignet erschien. ${ }^{11}$ Derartige Überlegungen kennzeichnen auch die Filme von Esfir Shub. So verdeutlichen beispielsweise die zaristischen Filmaufnahmen in DER FALI DER DYNASTIE ROMANOW, dass sich durch das Umschneiden von Einstellungsfolgen die Bedeutung von Bildern verändern und sich ihr ursprünglicher Sinn überschreiben lässt. Durch seine Montage akzentuiert der Film darüber hinaus die gesellschaftlichen Konflikte im vorrevolutionären Russland. Die Kombination der Aufnahmen evoziert Emotionen

10 Zur Gegenüberstellung der (politischen) Antagonisten als grundlegendes Prinzip des Films vgl. Leyda 1964, 27f; Guynn 2006, 147ff.

11 Vgl. Eisensteins Überlegungen zur «Dramaturgie der Film-Form» (1998 [1929]). 
oder abstrakte Begriffe, wobei Shub eine klare politische Zielrichtung verfolgt: den Zuschauern die Unausweichlichkeit der Revolution und die Notwendigkeit der sozialistischen Gesellschaftsordnung vor Augen zu führen.

Auch in den Filmen der Thorndikes wird die Montage für ideologische Zwecke eingesetzt. Die Filmemacher verlassen sich allerdings nicht auf die argumentative Kraft, die der ‘Zusammenprall〉 (Eisenstein) zweier Einstellungen erzeugt, sondern vereindeutigen die Bedeutung ihrer Bildkombinationen in der Regel durch einen dominanten Voice-over-Kommentar. Darüber hinaus betonen ihre Montagen kaum Konflikte, sondern operieren vor allem mit Vergleichen. In DU UND MANCHER KAMERAD findet sich beispielsweise eine Abfolge von Einstellungen, die eine Parallele zwischen verschiedenen marschierenden Formationen zieht. Zuvor wurde die militaristische Geschichte Deutschlands dargestellt, bis sich der Film am Ende der Gegenwart zuwendet und die beiden deutschen Staaten miteinander vergleicht. Beschreibt er zunächst eine winkend an Wilhelm Pieck vorbeidefilierende Menschenmenge als darin geeint, «den Irrweg, der zweimal ins Unglück führte, nie wieder zu gehen», verdeutlicht er anschließend sehr anschaulich, dass die BRD diesem Irrweg weiterhin folgt. Zu sehen sind verschiedene zeitgenössische Aufmärsche und Umzüge von Schützenvereinen und Traditionsverbänden, die der Film mit den Worten «so fing es doch schon zweimal an» und entsprechenden historischen Filmaufnahmen mit militaristischen Aufmärschen aus den Jahren 1912, 1929 und 1936 gleichsetzt. Diese Montage nimmt eine starke Semantisierung der aktuellen Bilder vor, wobei die Produktivität dieses Verfahrens darin besteht, dass der Film visuelle und inhaltliche (politische) Analogien für seine Argumentation (Wiederbewaffnung der BRD, personelle Kontinuität und Integration ehemaliger Nazis als zwangsläufige Bedrohung des Friedens) in eins setzt. ${ }^{12}$

Insgesamt ist die Argumentation von DU UND MANCHER KAMERAD um eine rationale Beweisführung bemüht. Sie akzentuiert beispielsweise in ihrer Erklärung der Kriegsursachen die Kausalität und logischen Zusam-

12 Zum «Grundmißverständnis» dieser rein «äußerlichen Analogie» vgl. Roth (1977, 172), der das Fehlen einer politischen Analyse bemängelt. Dieser Kritik lässt sich hinzufügen, dass der Film einige Minuten später die visuelle Ähnlichkeit isnoriert, die zwischen den historischen bzw. den westdeutsch Aufnert, die vom Aufmarsch der neu gegründeten Volksarmee besteht. Durch den Voice-overKommentar, die Musik sowie die Kombination mit historischen Aufnahmen von Verhaftungen oder von der gewaltsamen Auflösung friedlicher Demonstrationen sollen die Bilder von den NVA-Soldaten, die mit Sturmgewehren vor ihrer Brust an der Kamera vorbeimarschieren, nicht Krieg, sondern Verteidigung des Friedens signifizieren. menhänge von Kapitalismus und Krieg. Zwar operiert der Film auch mit Emotionen, die nicht zuletzt von Paul Dessaus Filmmusik evoziert werden, doch seine antikapitalistische Aussage und seine Kritik am bundesdeutschen Umgang mit der faschistischen Vergangenheit trifft der Film auf Basis rationaler Argumente, die vom Voice-over-Kommentar vorgetragen werden.

Unterscheidet sich auch das Montageverfahren und damit die Argumentationsform der Thorndikes deutlich von Esfir Shubs dialektischer Methode, so ähneln sich die Filme doch in ihrem Verständnis der Filmbilder als historische Quelle. Und wo Esfir Shub die dokumentarische Autorität der von ihr gefundenen Einstellungen verstärkt, indem sie diese langsam schneidet und dadurch den Zuschauern ermöglicht, sich in jede Einstellung zu vertiefen (vgl. Malitsky 2004), da lenken die Thorndikes die Aufmerksamkeit verbal oder mit visuellen Elementen (Pfeile) auf bestimmte Aspekte in den Filmbildern. Beiden Verfahren ist gemein, dass sie filmische oder fotografische Aufnahmen als Dokumente einsetzen, die Auskunft über die Vergangenheit geben. Die Filme der Thorndikes situieren diese Dokumente allerdings nicht nur im historischen, sondern auch im juridischen Diskurs. Vor allem in URLAUb AUF SYLT und UNंTERNEHMEN TEUTONENSCHWERT, die beide Bestandteil der Reihe ARCHIVE SAGEN AUS sind, werden sie zu Beweismaterial, um die NS-Belastung von bundesdeutschen Politikern und Offizieren nachzuweisen. ${ }^{13}$

\section{Die Filmreihe ARCHIVE SAGEN AUS}

Der Reihentitel ARCHIVE SAGEN AUS etabliert den diskursiven Rahmen, der die Argumentation und Rhetorik der Filme bestimmt. Dass es sich bei dem ‘Fall`, in dem die Archive mit ihren ‘Aussagen` für Aufklärung sorgen sollen, um den Nationalsozialismus handelt, macht bereits der Reihen-Vorspann deutlich, auf dessen Tonspur Ausschnitte aus Reden von Goebbels und Freisler, Heil-Rufe, die Fanfare der Wochenschau usw. zu hören sind. Zu dieser Kompilation von Tönen ist zunächst eine Filmdose des Reichfilmarchivs mit der Aufschrift «Geheim» zu sehen, dann gewährt die Kamera Einblick in ein Filmlager, indem sie durch die Gänge und an unzähligen Filmdosen vorbei fährt.

13 Die Reihe ARCHIVE SAGEN AUS umfasst mehrere zwischen 1957 und 1962 produzierte Filme, die auf eine Beeinflussung der Anti-Atomwaffen-Bewegung zielten, indem sie den in der BRD herrschenden «Militarismus` vor Augen führten. Nicht zuletzt die ungeordneten Bestände aus dem Moskauer Filmarchiv sollten hierfür das Beweismaterial liefern (vgl. Heimann 1996, 83). 
Der Vorspann legt damit die Erwartung nahe, dass es vor allem Filmaufnahmen sind, die in UrLaub auf Sylt und Unternehmen TeutonenSCHWERT als Beweismaterial für die Verbrechen der verdächtigten «Protagonisten dienen. Doch NS-Straftaten sind nur selten filmisch dokumentiert, und so ist es nicht verwunderlich, dass die beiden Filme vor allem auf Schriftdokumente und Zeugenaussagen zurückgreifen, um ihre Anklagen zu belegen, wobei durch die Verwendung juridischer Versatzstücke (z.B. Beglaubigungen und eidesstattlicher Erklärungen), deren Status als Beweismittel zusätzlich akzentuiert wird. Den Filmaufnahmen kommt demgegenüber oft eine illustrative Funktion $\mathrm{zu}$, insofern sie beispielsweise Deportationen oder den Rückzug der Wehrmacht im Allgemeinen, d.h. ohne Datierung oder andere konkretisierende Angaben, zeigen. Eine Tatbeteiligung der «Angeklagten` lässt sich mit diesen Bildern zwar nicht nachweisen, doch es entstehen - vom Kommentar unterstützt -visuelle Beweisketten, die entsprechenden Schlussfolgerungen Evidenz verleihen. Wirkt die geringe Ausbeute an juristisch verwertbaren Aufnahmen angesichts des umfangreichen Materialfundus, auf den die Filme zurückgreifen konnten, geradezu paradox, gelingt es ihnen doch, diese äußerst produktiv einzusetzen, um zu belegen, dass die faschistischen und militaristischen Netzwerke in der BRD fortbestehen und eine Bedrohung für den Frieden darstellen.

Schon Du und MANCher KAmERAd enthält eine Szene, in der diese Verfahren kurz zur Anwendung kommen: Der oben beschriebene Vergleich von Aufmärschen aus der Vorkriegszeit und zeitgenössischen Umzügen von Traditionsvereinen enthält auch aktuelle Aufnahmen vom Soldatenverein «Stahlhelm». "Stahlhelm marschiert», kommentiert die Voice-over diese Bilder, «an seiner Spitze: Kesselring». Nach einem kurzen Blick auf den Genannten, der hinter einem Rednerpult steht, folgt eine historische Aufnahme, die Hitler im Gespräch mit drei uniformierten Männern zeigt, wobei einer von ihnen durch einen dicken weißen Pfeil hervorgehoben wird. "Ganz richtig, jener Kesselring», identifiziert der Kommentar den Mann und wechselt mit diesem visuellen Beleg seiner Zugehörigkeit zur NS-Elite in den juristischen Diskurs: «Zum Tode verurteilt, freigekommen, auf allen Kriegsverbrecherlisten der Welt zu finden», fährt der Kommentar zu einer Einstellung fort, die festhält, wie Kesselring (vermutlich nach seiner Haftentlassung) von mehreren Männern herzlich begrüßt wird, und schlussfolgert: «Zum dritten Mal bereit.» Nach einer Aufzählung der Namen weiterer Wehrmachtsgenerale, die ebenfalls wieder «da» und «bereit sind», geht der Film auf zwei ranghohe Generale der neu aufgestellten Bundeswehr ein: Heusinger, «der in Hitlers Hauptquartier die Überfälle auf Europas Länder vorbereitete», und Speidel, «der die Taktik der ver-

1 Wieder «da» und «bereit»: Speidel und Heusinger

brannten Erde ausarbeitete». Nach der Einblendung von Fotos, auf denen die beiden jeweils mit Hitler zu sehen sind, folgt eine aktuelle Einstellung, die festhält, wie Adenauer mit Heusinger und Speidel eine Parade abnimmt, wobei die beiden Generale durch Pfeile hervorgehoben werden (Abb. 1).

«Diese beiden, Heusinger und Speidel, führen die westdeutsche Armee. Zum dritten Mal bereit», warnt der Kommentar und verdeutlicht diese Bedrohung durch Bilder der Bundeswehr und den Worten: «So fing es doch schon zwei Mal an.» Schließlich leitet er mit der erneuten Feststellung, «zum dritten Mal in ihrer Gewalt», dazu über, seine Frage, «wie ist das möglich?», mit den unveränderten Besitzverhältnissen des Industriekapitals in der BRD zu beantworten.

In dieser kurzen Szene aus Du UND MANCHER KAMERAD deutet sich bereits die Methode an, die dann in den beiden Filmen aus der Reihe ARCHIVE SAGEN AUS systematisch weiterverfolgt wird. Alle ThorndikeFilme über die nationalsozialistische Vergangenheit weisen einzelnen bundesdeutschen Funktionsträgern die Beteiligung an NS-Verbrechen nach. Durch die Verknüpfung von aktuellen und historischen Aufnahmen entsteht eine visuelle Beweiskette für diese personelle Kontinuität. Genügt in Du und MANCHer Kamerad jedoch jeweils ein einziges Foto mit Hitler und dem Beschuldigten als Beleg für dessen NS-Belastung, so führen die beiden anderen Filme eine Vielzahl weiterer Dokumente ins Feld, um ihre Anklage zu untermauern. Während die Verantwortung einzelner Personen in Du Und MANCher Kamerad damit vor allem eine Behauptung bleibt, für die der Film - bis auf die fotografisch festgehaltene (räumliche) Nähe zu Hitler - keine weiteren Belege liefert, präsentieren URLAUb AUF SYLT und UNTERNEHMEN TeUtonensChWERT ihr Beweismaterial in aller Ausführlichkeit und unterstreichen dadurch 


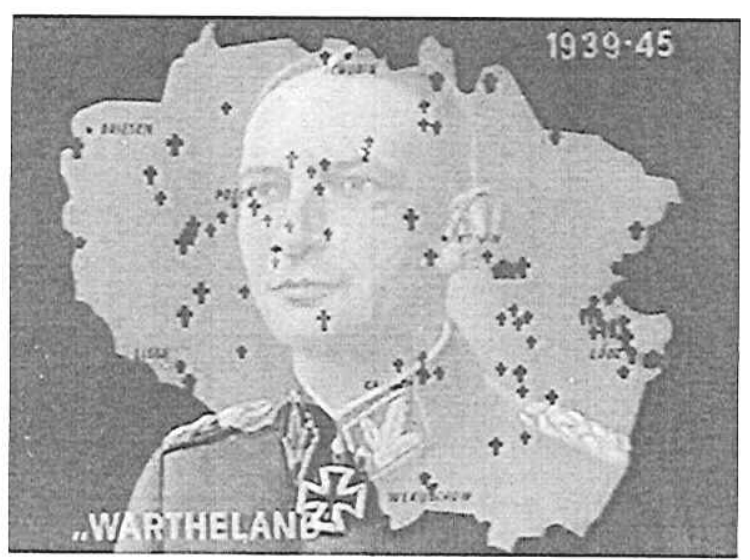

2 Reinefahrt als Verantwortlicher für NSVerbrechen

die Glaubwürdigkeit ihrer Aussagen und die Nachvollziehbarkeit ihrer Anklagen.

URLAUB AUF SYLT beginnt wie ein Werbefilm für die Urlaubsinsel, in dessen Rahmen auch dem amtierenden Bürgermeister von Westerland ein Besuch abgestattet wird. "Weitab von der großen Welt und unbeachtet von ihr", so eine weibliche Kommentarstimme in betont konventioneller Werbefilm-Intonation, "versieht Herr Reinefarth sein Amt», worauf eine männliche Stimme mit ähnlicher Diktion anschließt: «Aber wer Herrn Reinefarth näher kennt weiß, der Bürgermeister von Westerland ist zu Unrecht unbeachtet von der Welt. Denn nicht immer war Herr Reinefarth Bürgermeister.» Daraufhin wird das Filmbild angehalten und die Porträtaufnahme von Reinefarth in ein älteres Foto überblendet, das dem Schrvarzen Korps, der Wochenzeitung der SS, entnommen ist, die über die Verleihung des Ritterkreuzes mit Eichenlaub an Reinefarth berichtet. Neben dieser Überblendung, die hier als visuelle Metapher fungiert, mit der URLAUb AUF SYLT die Kontinuität der alten Machteliten akzentuiert (und diese sehr viel deutlicher vor Augen führt als mit einem abrupten Einstellungswechsel), weist der Film Reinefarths Verantwortung für NSVerbrechen anhand verschiedener Dokumente nach. Beispielsweise werden Schriftstücke gezeigt, in denen Reinefarth von Himmler zum Höheren SS- und Polizeiführer des SS-Oberabschnitts Warthe ernannt und später von Hitler selbst zum SS-Gruppenführer und Generalleutnant der Polizei befördert wurde. Diese Dokumente verknüpft der Film mit Fotos von Erschießungen und Erhängungen, die im Warthegebiet stattgefunden haben. Dabei unterstreicht er die Verantwortlichkeit Reinefarths wiederum durch ein visuelles Stilmittel, diesmal durch eine Doppelbelichtung, die Reinefarths Porträt über eine Karte einblendet, in der Massengräber verzeichnet sind (Abb. 2).

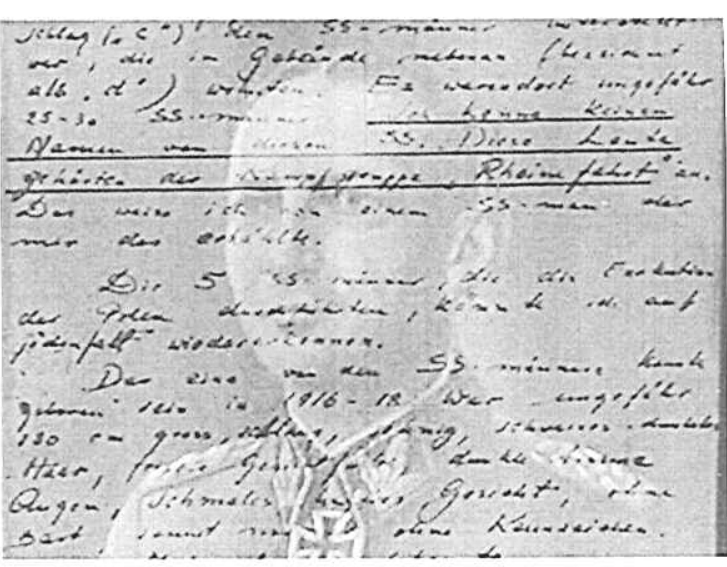

Schriftliche Aussage gegen Reinefahr

Die besondere Aufmerksamkeit von UrLAUb AUF SYLT gilt dem Aufstand der Stadt Warschau 1944. Zunächst schildert der Film die Situation in der Stadt und die mutigen Vorbereitungen für den Aufstand, dann ist ein Ausschnitt aus der Deutschen Wochenschau zu sehen, die von den Kämpfen in der Stadt berichtet und dazu Bilder der Zerstörung zeigt. Schließlich kommt URLAUB AUF SYLT auf Reinefarth zurück, dem die Gesamtführung der SS-Verbände zur Niederschlagung des Aufstandes anvertraut war. Der Film belegt diese Funktion durch ein abgefilmtes (und mit einem aktuellen Porträt überblendeten) Schriftdokument und präsentiert dann die Aussage eines deutschen Unteroffiziers in Kriegsgefangenschaft, der Reinefarths Verantwortung für die Massenexekutionen von Zivilisten bei der Niederschlagung des Aufstands bezeugt. Der Augenzeuge berichtet von systematischen Erschießungen, die Angehörige der SS-Gruppe Reinefarth verübt haben. Ganz der Rhetorik des juristischen Diskurses entsprechend, nennt URLAUB AUF SYLT zunächst den Namen und militärischen Rang dieses Zeugen sowie das Datum seiner Aussage, wodurch diese besonderes Gewicht erhält. Während der Voice-over-Kommentar vorliest, was der ehemalige Soldat zu Protokoll gab, ist im Bild das handschriftliche Dokument seiner Aussage zu sehen (wobei zur Hervorhebung und zum Mitlesen einzelne Passagen unterstrichen werden) (Abb. 3) und eine Skizze, mit deren Hilfe UrLAUB AUF SYLt die Vorgänge rekonstruiert. Darüber hinaus werden auch Fotos von Erschießungen gezeigt.

UrLAUb AUf Sylt warnt vor der Gefahr, die von der BRD ausgeht: Der von CDU und BHE (Bund der Heimatvertriebenen und Entrechteten) zum Bürgermeister gewählte NS-Verbrecher Reinefarth erwarte «auf Sylt den Ruf zu neuem Einsatz», so der Film, der zeigt, dass sich die calte Garde bereits neu formiert hat und zum Sammeln trommelt. Zu sehen sind Auf- 


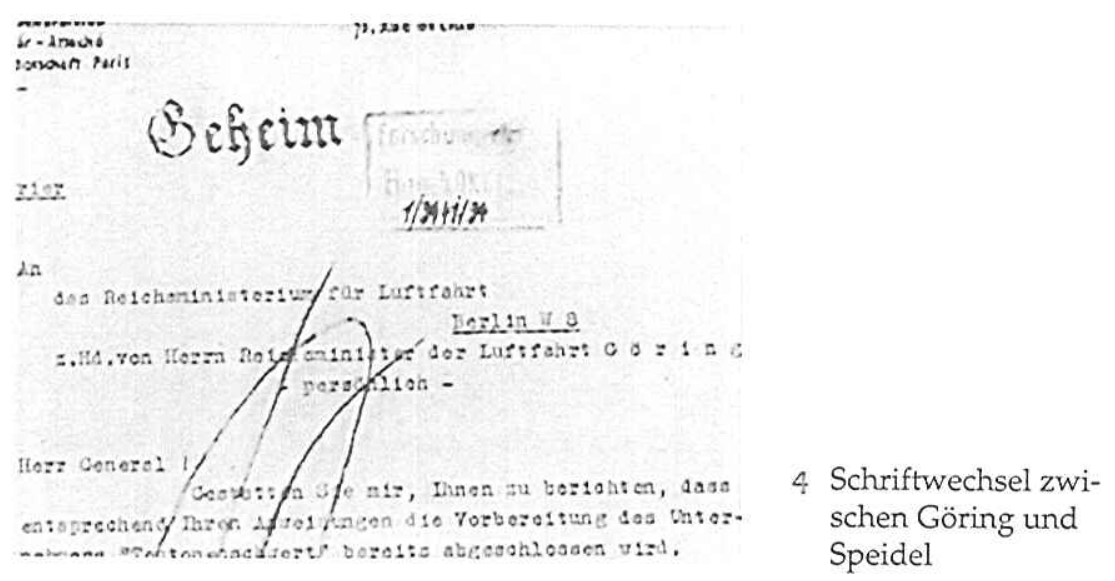

nahmen von einem Treffen des ‘Traditionsverbandes' HIAG (Hilfsgemeinschaft auf Gegenseitigkeit der ehemaligen Angehörigen der Waffen-SS), auf dem die Teilnehmer unter anderem Kurt Meyer (,Panzermeyer $`$ ) feiern, der in Kanada aufgrund seiner Kriegsverbrechen zum Tode verurteilt, durch die Intervention mehrerer bundesdeutscher Persönlichkeiten jedoch aus der Haft entlassen worden war. Mit dem Hinweis, dass inzwischen «unzählige große und kleine Panzermeyers und Reinefarths» die "Kommandostellen» in Westdeutschland besetzt halten und nur auf einen neuen Einsatz warten, sowie der Schrifttafel «Menschen seid wachsam» endet der Film.

Belegen die aktuellen Aufnahmen vom Treffen ehemaliger SS-Leute eindrücklich das ungebrochene (und offiziell anerkannte) Fortbestehen nationalsozialistischer Netzwerke in der BRD, so mangelt es URLAUB AUF SyLt an historischen Aufnahmen von Reinefarth - und damit auch an fotografischem Beweismaterial für dessen Verbrechen. Implizit thematisiert der Film dies selbst, wenn er im Zusammenhang mit Reinefarths Tätigkeit in Warschau darauf hinweist, dass die Niederschlagung des Aufstands kaum visuell dokumentiert ist: «Von diesem ihrem Einsatz in Warschau, Herr Reinefarth», wendet sich die Kommentarstimme direkt an den Beschuldigten und unterstreicht damit ihre Rolle als Ankläger, «sind der Nachwelt nur einige wenige Bilder erhalten geblieben». Daraufhin präsentiert der Film diese Fotografien und Filmaufnahmen, die zerstörte Häuser, festgenommene Menschengruppen und zahlreiche Leichen, nie jedoch Reinefarth zeigen. Dennoch schließt sich der lose Zusammenhang von Bildern und Anklage zu einer Beweiskette. Es entsteht der Eindruck, URLAUB AUF SYLT liefere den Nachweis über Reinefarths Verbrechen anhand historischer Filmaufnahmen, obwohl es letztendlich die Zeugenaussage

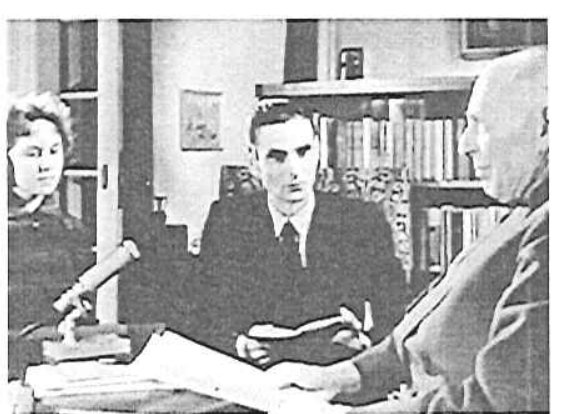

5 Aufklärung des Zeugen über strafrechtliche Folgen einer Falschaussag

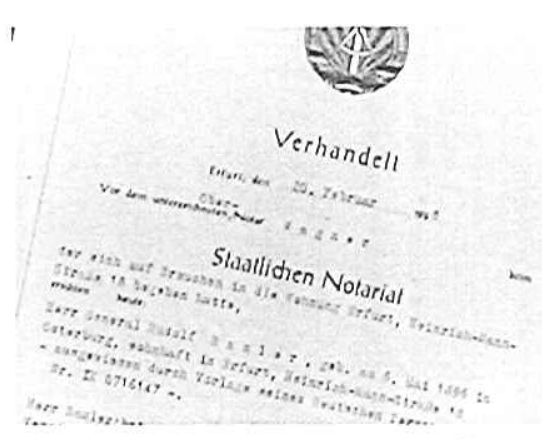

6 Notarielle Beglaubigung der Zeugenaussage und die Schriftdokumente sind, deren juristische Beweiskraft der Anklage Glaubwürdigkeit verleiht. ${ }^{14}$

Ähnlich verfährt auch UNTERNEHMEN TeUtONENSCHWERT, in dem NATO-General Hans Speidel im Mittelpunkt steht, wobei sich die Rekonstruktion seiner Vergangenheit zeitweise als Aufdeckung einer Verschwörung darstellt. ${ }^{15}$ Der Film beginnt mit dem Attentat auf Körig Alexander von Jugoslawien und den französischen Außenminister Louis Barthou im Oktober 1934. Der jugoslawische König war nach Marseille gereist, um einen Bündnisvertrag zu unterzeichnen, mit dem Frankreich dem Expansionsbestreben Deutschlands entgegenwirken wollte. UNTERNEHMEN TEUTONENSCHWERT weist nun anhand einer Reihe von Geheimdokumenten nach, dass Hans Speidel als Mitarbeiter des Militärattachés der deutschen Botschaft in Paris an der Planung dieses Attentats beteiligt war. Um dies zu belegen, zitiert der Film aus dem (geheimen) Schriftverkehr zwischen Göring und Speidel. Währenddessen sind die entsprechenden Dokumente auch auf der Bildebene zu sehen (Abb. 4).

Doch UnTERNEHMEN TeUtONENSCHWERT zieht nicht nur Schriftdokumente für seine Beweisführung heran. Speidels Spionagetätigkeit wird auch von einem Zeugen bestätigt, dessen eidesstattliche Aussage der Film ausführlich dokumentiert: In einer gut vierminütigen Szene gibt $\mathrm{Ru}-$

14 Heimann $(1996,83)$ und Steinle $(2003,139)$ weisen darauf hin, dass der Film für Reinefarth keine Konsequenzen hatte (dieser ist erst 1964 aufgrund von Ermittlungen wegen Kriegsverbrechen von seinem Amt als Bürgermeister zurückgetreten), das westdeutsche Kamerateam, das Reinefarth in Westerland gefilmt hatte, jedoch wegen "Spionagetätigkeit» zu sechs Monaten Gefängnis verurteilt wurde.

151962 gewann Hans Speidel eine Klage gegen die britische Verleihfirma, weil die Echtheit einiger Dokumente nicht nachgewiesen werden konnte (vgl hierzu Steinle 2003 , heit einiger Dokumente nicht nachgewiesen werden konnte (vgl. hiezu Steinle 2003, 140ff). Spätere Gutachten, die im Auftrag des Münchener Instituts für Zeitgeschichte und des Auswärtigen Amts der BRD erstellt wurden, bestätigten die Manipulation de Dokumente; vgl. hierzu Heimann 1996, 90, Fußnote 68. 


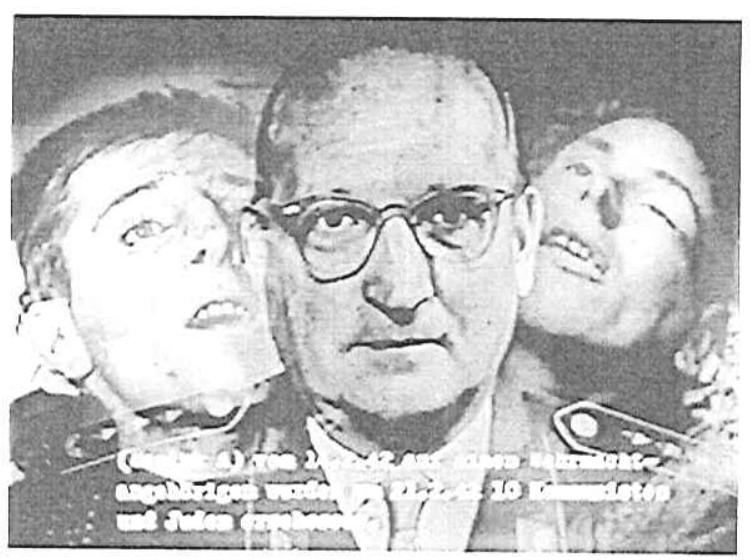

7 Speidel als Verantwortlicher für Vergeltungsmaßnahmen

dolf Bamler, ehemaliger Generalleutnant der Abwehr, der in sowjetischer Kriegsgefangenschaft dem «Nationalkomitee Freies Deutschland» beigetreten war und einen Aufruf gegen Hitler unterzeichnet hatte, zu Protokoll, dass Speidel eine Schlüsselposition in der deutschen Spionage innehatte. Ihre besondere Autorität erhält diese Aussage dadurch, dass der Film auch das notarielle Prozedere dokumentiert, das der eigentlichen Aussage voranging und ihre Glaubwürdigkeit besonders unterstreicht. $\mathrm{Zu}$ sehen ist ein Notar mit seiner Mitarbeiterin, der Bamlers Personalien aufnimmt und ihn unter Nennung des entsprechenden Paragrafen im Strafgesetzbuch darüber aufklärt, dass eine wissentlich falsche Aussage mit einer Freiheitsstrafe bis zu drei Jahren bestraft wird (Abb. 5). Nachdem Bamler, der von unterschiedlichen Kamerapositionen aus gefilmt wird, seine zuvor niedergeschriebene Zeugenaussage verlesen hat, schließt die Sequenz mit dem Schriftdokument der notariellen Beglaubigung (Abb. 6).

Auch im Weiteren operiert der Film mit eidesstattlichen Erklärungen und Schriftdokumenten, um seine Thesen zu belegen. So verliest eine Kommentarstimme Passagen aus der Erklärung, die Manfred Rommel zum Selbstmord seines Vaters abgegeben hat, um anhand dieser Zitate nachzuweisen, dass Speidel Verrat an Erwin Rommel begangen habe. Erneut wird der juridische Diskurs akzentuiert, indem der Film von der Aussage eines «Kronzeugen» spricht und am Ende festhält, dass «Rommels Sohn Manfred den Schwur leistet». Während die entsprechende Stelle im abgefilmten Schriftstück durch Unterstreichung hervorgehoben wird, zitiert eine jugendliche Stimme: «Ich erkläre hiermit an Eides statt vor Zeugen, dass die Angaben auf Wahrheit beruhen - Manfred Rommel.»

Neben diesen Zeugenaussagen greift Unternehmen Teutonen-

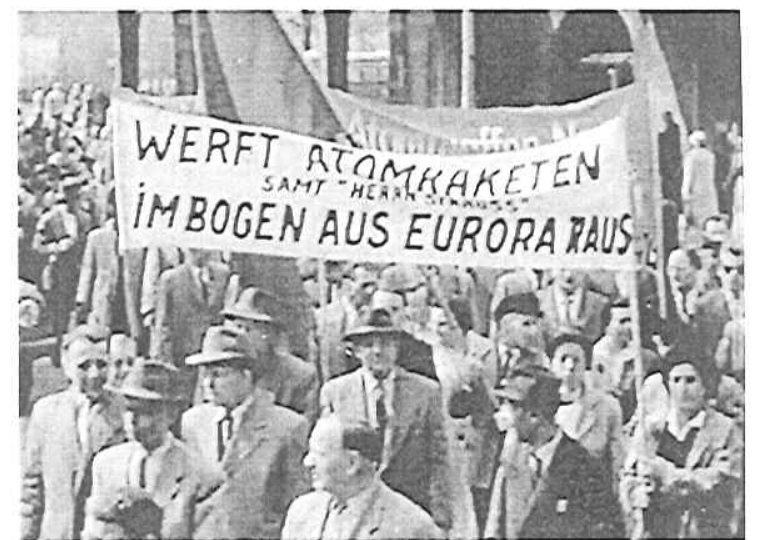

Demonstration gegen Atomwaffen

SCHWERT auch auf weitere Schriftdokumente zurück; so zitiert der Film aus dem Bericht «Stimmung und innere Sicherheit‘, den Speidel für den Oberkommandierenden der Wehrmacht über das besetzte Frankreich verfasst hat. Zuerst wird dieses Dokument authentifiziert, indem sein $\mathrm{Ab}$ fassungsdatum, der Eingangsstempel und das Signum Keitels präsentiert werden, dann liest eine männliche Voice-over einzelne Passagen aus dem Bericht vor, die im Bild zuerst durch Unterstreichung und dann durch die Einblendung eines Rolltextes hervorgehoben werden. Dabei handelt es sich um eine Auflistung von "Vergeltungsmaßnahmen», die im besetzten Frankreich befohlen worden waren. Während der Text noch vorgelesen wird, zeigt der Film eine aktuelle Aufnahme von Speidel, die mit Fotos von Leichen überblendet wird (Abb. 7). Legt der Film Speidels Schuld am Tod französischer "Kommunisten und Juden» hier nur visuell nahe, so weist ihm der Kommentar etwas später explizit die Verantwortung für die Taktik der (verbrannten Erde) zu, indem er Aufnahmen ankündigt, «die zeigen, wie Speidel den Rückzug der Achten Armee vollziehen ließ». Ähnlich wie in URLAUB AUF SYLT wird hier ein loser Zusammenhang filmisch zu einer Beweiskette verknüpft, wobei UNTERNEHMEN TEUTONENSCHWERT die Kriegsbilder auch für die Darstellung des gegenwärtigen Bedrohungsszenarios produktiv macht. Mit der Bemerkung, "das richtete der Hitler-General Speidel mit konventionellen Waffen an», und der Frage, «was würde der NATO-General Speidel heute anrichten mit Atomwaffen?», erweckt der Film den Eindruck, dass von Speidel eine erhebliche Kriegsgefahr ausgeht.

Dieses Bedrohungsszenario verstärkt Unternehmen TeutonenSCHWERT durch die Aufzählung weiterer Personen, die im (Dritten Reich) zur militärischen oder rüstungsindustriellen Elite gehörten und in der BRD erneut wichtige Funktionen im Militär (Ruge, Kammhuber, Heu- 
singer) oder in der Wirtschaft (Messerschmitt, Krupp, Rohland, Werner, Geilenberg, Fischer, Frydag, Heyne) innehaben. Auch die Nennung ehemaliger SS-Offiziere, die in Traditionsverbänden oder als Verleger ihr Gedankengut weiterhin ungehindert verbreiten, verstärkt die These einer militaristischen BRD. Ähnlich wie DU UND MACHER KAMERAD (und teilweise mit Bildern, die bereits in URLAUB AUF SYLT zu sehen waren) unterstreicht der Film die personellen Kontinuitäten, indem die NSVergangenheit der jeweiligen Person anhand von historischen Aufnahmen belegt und diese dann mit aktuellen Aufnahmen verknüpft werden. Nach einer Einstellung von einer "Atomkanone», die der Film mit den Worten «alle, die wir Euch zeigten, warten darauf, sie einzusetzen», kommentiert, endet der Film schließlich mit dem Hinweis, «diese Kanone kann unser Leben auslöschen. Aber wir können auch diese Kanone auslöschen", und Aufnahmen von einer großen Friedensdemonstration, zu denen das Anti-Atomwaffen-Lied «Wir wollen Frieden auf lange Dauer» zu hören ist (Abb. 8).

Wie URLAUb AUF Sylt enthält auch UnTERNEHMEn TeUtonensChwert keinen fotografischen oder filmischen Beleg für die kriminellen Handlungen seiner Hauptfigur. ${ }^{16}$ Beide Filme nutzen für ihre (visuelle) Beweisführung daher Schriftdokumente und Zeugenaussagen. Durch die spezifische Kommentierung des Bildmaterials sowie die Montage und Überblendung der vorhandenen Aufnahmen von den Protagonisten mit anderem Beweismaterial gelingt es den Filmen jedoch, den Eindruck eines visuellen Nachweises zu erwecken.

Angesichts der umfangreichen Materialrecherche, die Andrew und Annelie Thorndike betrieben haben, nimmt sich die Ausbeute an Filmaufnahmen, die sich für ihre Auseinandersetzung mit dem bundesdeutschen Verhalten zur nationalsozialistischen Vergangenheit als Beweismittel produktiv machen lassen, äußerst mager aus: Zwar haben die Beiden wichtige Filmdokumente (z.B. Gestapo-Aufnahmen von ausgemergelten Kindern im Warschauer Ghetto [vgl. Heimann 1996, 75]) zu Tage gefördert; ihre spärlichen Entdeckungen im Fall Reinefarth und Speidel wirken jedoch

16 Im Unterschied zu UrLaub auf Sylt kann Unternehmen Teutonenschwert jedoch immerhin auf eine Filmeinstellung von Speidel zurückgreifen: Eine Halbtotale zeigt Himmler, der durch ein Fernglas blickt, er ist so platziert, dass er eine Reihe weiteer, neben ihm stehender Manner verdeckt. Einer dieser Manner beugt sich jedoch etwas nach vorne und blickt in Richtung Kamera. Diesen identifiziert der Film als Han Speidel. Um ihn besser kenntlich zu machen, wird ein großer Pfeil eingefügt und das Filmbild sogar für einen Augenblick angehalten. Mit diesem tricktechnischen Eingriff und der Behauptung, Speidel habe die Kamera immer gemieden, gibt UNTERNEHMEN TEUTONENSCHWERT vor, einen weiteren Beweis für dessen geheime Unternehmungen liefern zu können. wie ein Eingeständnis der Tatsache, dass sich historische Aufnahmen sehr gut zum Erzählen von Geschichte(n), nicht jedoch als juristisches Beweismaterial eignen.

\section{Geschichtspolitik}

Bereits der Titel ArChIve SAgEn Aus macht deutlich, dass sich diese Reihe im juridischen Diskurs positioniert. Diesem Selbstverständnis entsprechend werden in den Filmen (pseudo-)juristische Beweismittel herangezogen, auf deren Grundlage sie am Ende ein Urteil fällen. In URLAUB AUF SYLT und UNTERNEHMEN TEUTONENSCHWERT sind es vor allem Schriftdokumente und Belastungszeugen, mit denen die Filme den sangeklagtens Personen eine Beteiligung an kriminellen Handlungen während der Zeit des Nationalsozialismus nachweisen. Die Verwendung von juristisch geprägten Begriffen (z.B. «Kronzeuge») und die direkte Adressierung der Protagonisten, mit denen eine Vernehmungssituation simuliert wird, verstärken zusätzlich den Rahmen des juridischen Diskurses. Gleichzeitig bemühen sich die Filme, mit Hilfe der oben beschriebenen Verfahren die Tatbeteiligung der ‘Angeklagten` auch für die Zuschauer evident zu machen.

Mag es heute auch ungewöhnlich erscheinen, dass sich Filme solcherart positionieren, so war zur Entstehungszeit der besprochenen Beispiele dieser diskursive Rahmen für die Beschäftigung mit den Nationalsozialismus keine Seltenheit. Sowohl in der DDR als auch in der BRD fand in jener Zeit die Auseinandersetzung mit der Vergangenheit vor allem durch die juristische Strafverfolgung statt. Die beiden deutschen Staaten gingen dabei jedoch sehr unterschiedlich zu Werke: Galten in der SBZ «einfache NSDAP-Mitglieder und andere ‘Mitläufer» als entnazifiziert, wenn sie sich aktiv in den neuen sozialistischen Staat integrierten, wurden demgegenüber die ehemaligen Machteliten strafrechtlich hart verfolgt. Diese setzten sich daher nach Westdeutschland ab, wo die Gefahr eines Ermittlungsverfahrens wegen NS-Straftaten relativ gering war. Gegen diese Personengruppe, die juristisch nicht zur Verantwortung gezogen worden war und in der bundesdeutschen Gesellschaft schnell wieder Fuß gefasst hatte, richtete sich die Anklage der Filme. Mit ihrer Kritik an der westdeutschen (juristischen) Aufarbeitung des Nationalsozialismus sind die Filme Bestandteil einer Geschichtspolitik, in deren Rahmen sich die DDR aufgrund ihres Antifaschismus als das moralisch bessere Deutschland definierte und damit gleichzeitig eine Idealisierung der eigenen Gesellschaft betrieb (vgl. Herbert/Groehler 1992). 
Aus historischer Perspektive lassen sich die Filme der Thorndikes als Dokumente ihrer Entstehungszeit verstehen und im Kontext der deutschdeutschen Beziehungen bzw. des Kalten Krieges diskutieren. Gleichzeitig stellen die Filme aus der Reihe ARCHIve SAGEN AUS auch heute noch Informationen bereit, die bei der gesamtdeutschen Vergangenheitsbewältigung gerne in Vergessenheit geraten. Sie erinnern daran, dass die Bewertung des Nationalsozialismus lange Zeit umkämpft war, und vermögen Verwunderung darüber hervorzurufen, dass die BRD trotz ihrer Integration der nationalsozialistischen Eliten zu einer stabilen Demokratie wurde.

\section{Literatur}

Eisenstein, Sergej M. (1998) Dramaturgie der Film-Form. Der dialektische Zugang zur Film-Form [1929]. In: Texte zur Theorie des Films. 3. durchges. u. erw. Aufl. Hg. v. Franz-Josef Albersmeier. Stuttgart: Reclam, S. 275-304.

Guynn, William (2006) Writing History in Film. New York/London: Routledge.

Heimann, Thomas (1996) Von Stahl und Menschen 1953 bis 1960. In: Schrvarzweiß und Farbe. DEFA-Dokumentarfilme 1946-1992. Red. Günter Jordan \& Ralf Schenk. Berlin: Jovis, S. 49-91.

Herbert, Ulrich / Groehler, Olaf (1992) Zweierlei Bewülttigung. Vier Beiträge über den Umgang mit der NS-Vergangenheit in den beiden deutschen Staaten. Hamburg: Ergebnisse-Verlag.

Leyda, Jay (1964) Films Beget Films. London: Allen \& Unwin. [Dt. als: Filme aus Filmen. Eine Studie ïber den Kompilationsfilm. Berlin: Henschel 1967].

Malitsky, Josh (2004) Esfir Shub and the Film Factory-Archive. Soviet Documentary from 1925-1928. In: Screening the Past, Nr. 17 [http:/ / www.latrobe.edu.au/ screeningthepast/firstrelease/fr_17/JMfr17a.html].

Roth, Wilhelm (1977) Dokumentaristen. Wege zur Wirklichkeit. In: Film in der $D D R$. Hg. v. Stiftung der deutschen Kinemathek, in Zusammenarbeit mit Peter W. Jansen \& Wolfram Schütte. München: Hanser, S. 167-202.

Steinle, Matthias (2003) Vom Feindbild zum Freundbild. Die gegenseitige Darstellung von BRD und DDR im Dokumentarfilm. Konstanz: UVK.

Vonau, Ingolf (2002) Das 70-mm-Filmformat in der DDR. Technologie, Entwicklung, Projekte: Anspruch und Realität. In: Aufstieg und Untergang des Tonfilms. Hg. v. Joachim Polzer. Potsdam: Polzer Media Group, S. 423-439 (Weltwunder der Kinematographie, Bd. 6).

Wees, William C. (1992) Found Footage und Fragen der Repräsentation. In: Found Footage Film. Hg. v. Cecilia Hausheer \& Christoph Settele. Luzern: Viper/Zyklop, S. 36-52.

\section{Quellen}

DEFA-Stiftung: Online-Datenbank des Filmbestands (www.defa-stiftung.de) Filmportal (www.filmportal.de)

Friedrich-Wilhelm-Murnau-Stiftung: Online-Datenbank des Filmbestands (www. murnau-stiftung.de)

Programmtexte des Kinos Arsenal (www.arsenal-berlin.de/arsenal/archiv.html) 